\title{
A further study of the partial reinforcement effect within-subjects'
}

\author{
WILLIAM B. PAVLIK, PETER L. CARLTON AND PETER G. MANTO
}

RUTGERS UNIVERSITY

\begin{abstract}
The effects of partial and continuous reinforcement schedules upon subsequent resistance to extinction were studied, within-subjects, in a lever-pressing situation. Previous research with this design had yielded a reversed partial reinforcement effect. The training procedures of the present experiments were such that a switch in schedules occurred only after a given number of responses or reinforcements had occurred in the previous schedule. The results suggest a "preference" for continuous reinforcement during training, and clearly yield a conventional partial reinforcement effect during extinction.
\end{abstract}

\section{Introduction}

The conventional partial reinforcement effect (PRE) is that animals trained on a partial reinforcement (PR) schedule emit more responses in extinction than do animals trained on a continuous (CRF) schedule. This result has typically been obtained by comparing two groups, one trained on PR, the other on CRF.

In a recent study (Pavlik \& Carlton, 1965), we compared the effects of PR and CRF schedules in a leverpressing situation in which one group of animals was exposed to both schedules. We used a multiple schedule in which one component was CRF, the other VR3. Control groups exposed to only one of the schedules were also studied.

We found that, in extinction, animals that had been exposed to both schedules emitted more responses in the stimulus associated with CRF than in the stimulus associated with VR3; a reversed PRE. A conventional PRE was obtained from the comparison of the control groups.

One methodological flaw in that study was that neither numbers of responses nor numbers of reinforcements were equated for the two components of the multiple schedule; the animals were given the same amount of time in both schedules. The present study examined the PRE within-subjects when either responses or reinforcements during training had been equated.

\footnotetext{
Method

Subjects. The Ss were 12 experimentally naive male Sprague-Dawley rats which weighed between 140 and $160 \mathrm{gm}$ at the start of the experiment.

Apparatus. The apparatus was a two-lever Grason-Stadler response chamber, appropriately programmed, and equipped with a pelletdispenser for $45 \mathrm{mg}$ Noyes pellets.

Procedure. The animals were placed on a feeding schedule $(10 \mathrm{gm}$ of Lab Chow per day) four days prior to the start of training. Water was always available in the home cage. Following magazine training, the animals were given one day of bar training in which responses to the right-hand lever were reinforced (CRF) until 50 reinforcements had been taken. On the following day, Day 2, the lights over the levers were alternately lighted (30 sec. alternation) and responses to the lighted lever (only) were reinforced until another 50 reinforcements had been taken.
}

On the following day, 6 animals were assigned to Experiment 1 and the remaining 6 to Experiment 2. For the animals in Experiment 1, the procedure for the following 9 days was the same as for Day 2, with two exceptions. The position of the lighted lever switched after 10 responses had been made to the lighted lever, and one of the levers produced reinforcements on a VR3 schedule; the other lever remained on CRF. For 3 animals, the right-hand lever was the CRF lever; the left-hand lever was on CRF for the remaining 3 animals. Each daily session lasted until the animal had emitted 60 responses to each lighted lever, a procedure that produced 60 reinforcements on the CRF lever and approximately 20 on the VR3 lever.

For the 6 animals in Experiment 2, the training procedure was identical to that in Experiment 1, except that the position of the lighted lever switched after 10 reinforcements had been taken on the lighted lever, and that each session lasted until 40 reinforcements had been taken on each schedule. Thus, Experiment 1 equated for the number of responses made to the two levers, and Experiment 2 equated for the number of reinforcements taken on the two levers.

Following the 9 days of training, each animal was given two daily extinction sessions. The animals in the two experiments were treated alike during extinction. Each extinction session lasted 20 min., during which time the position of the lighted lever alternated each $30 \mathrm{sec}$.

Throughout both training and extinction, records were kept of the numbers of responses to the two levers, the total time each lever was lit, and the summed latency, measured as the time from the onset of a cue light to the first response to the appropriate lever.

\section{Results}

The principal results are summarized in Fig. 1, which presents, for both experiments, mean response rate in both schedules for the last day of acquisition and total responses in both schedules for the two days of extinction.

In each experiment, 5 of the 6 animals had higher rates during the last training session on the VR than on the CRF schedule. Every animal in both experiments also showed a considerably longer latency in commencing to press on the VR than on the CRF schedule. Mean summed starting latencies for the CRF and VR schedules were $1.6 \mathrm{sec}$. and $13.4 \mathrm{sec}$. in Experiment 1; in Experiment 2, they were 3.2 and $19.9 \mathrm{sec}$. When these differences are taken into account in computing response rates (the upper portions of the bars at the left of Fig. 1), the differences between the schedules are magnified, and only one animal (in Experiment 2) failed to show superiority of VR over CRF rate. Thus while an animal takes longer to start pressing on the VR schedule, his rate, once started, is

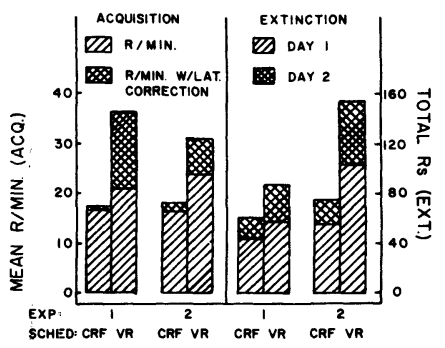


higher than on CRF。

As far as Experiment 1 is concerned, these rate and latency differences are, in part at least, a necessary consequence of the procedure. Because responses were equated, the animals necessarily spent more time in consuming the pellets during CRF than VR. This could have, of course, contributed to the lower rates in CRF. Furthermore, the switch in schedules was always concurrent with pellet delivery when the switch was from CRF to VR but was concurrent one-third of the time when the opposite switch was made. This factor would necessarily inflate the starting latencies in VR relative to those in CRF。

It is important to note, however, that neither of those factors could operate in Experiment 2 because, first, reinforcements in each schedule were equated and sec- $^{-}$ ond, all schedule-switches were concurrent with pellet delivery. Furthermore, unsystematic observation of the animals in Experiment 1 indicated that the time spent consuming a pellet was quite brief. This fact, and especially the comparability of the data from Experiments 1 and 2, make it very unlikely that the differences in rate and starting latency in Experiment 1 were entirely due to the contamination of differential pellet delivery between the schedules.

In addition to the rate and latency data, there was a third differential effect of schedule. When incorrect responses (responses made to a lever when that lever was not illuminated) during the final training session are considered, 5 of the 6 animals in each experiment made more incorrect responses on the CRF than on the VR lever, although the number of such responses was small in all cases. When the data of both experiments are pooled, a total of 44 incorrect responses were made to the CRF lever, and only 7 to the VR lever. Thus, both the starting latency and incorrect responses data suggest a "preference" for the CRF lever.

The extinction data, summarized on the right of Fig. 1, clearly indicate a conventional PRE in both experiments. On the first day of extinction, only one animal (in Experiment 1) emitted more responses to the CRF than to the VR lever. On the second day, and on both days pooled together, all animals in both experiments emitted more responses to the VR than to the CRF lever. For both extinction sessions pooled together, the differences between responses to the two levers ranged from 8 to 50 responses for individual animals in Experiment 1, and from 26 to 119 responses in Experiment 2. Similarly, all animals but one (in Experiment 1) emitted more incorrect responses to the VR lever than to the CRF lever; mean incorrect responses to the CRF and VR levers were 11.7 and 29.2, respectively, for Experiment 1 animals, and 6.5 and 68.0, respectively, for Experiment 2 animals. There were no differences in starting latencies during extinction, either between schedules or between experiments. During extinction, Experiment 2 animals did, however, emit both more correct and more incorrect responses to the VR lever than did Experiment 1 animals ( $\mathrm{p}<.05$ for both comparisons).

\section{Discussion}

The extinction procedures used herewe .G those used in the earlier study by Pavlik \& Carıun (1965); only the training procedures differed. In our earlier experiment, a reversed PRE was found, whereas the conventional effect was obtained here. In both experiments, large order but opposite effects were obtained.

In our earlier study, the reversed PRE was due to a suppression of extinction responses in the VR component of the multiple schedule (relative to the response levels of animals exposed only to VR).A related "preference" for CRF was obtained in the training phase of the present experiments; the animals took longer to start responding and made more incorrect responses in the VR component. This "preference" was not reflected in extinction, however. The basis for this lack of effect may have been that total responses or reinforcements had been matched during training by requiring a given number of responses or reinforcements prior to a switch in schedules. This could have led to the development of a response chain that obscured the effect seen in our earlier experiment.

In particular, a change in schedule during training in the present experiments was always contingent upon a preceding response. If the animals did "prefer" CRF (and internal evidence suggests that they did), then responding in the VR component would be secondarily and preferentially reinforced by the ensuing switch to CRF。 During extinction, then, performance in the VR component would be maintained not only by its prior reinforcement history but also by the change to a preferred schedule. The latter factor would not be effective in maintaining performance in the CRF component. (This difference did not appear in the summed latencies. This lack of difference may have been due to pooling of all values in the course of each extinction session; unfortunately, moment-to-moment values were not available.)

The conventional PRE observed in the present experiments may have been a reflection of the "preference" for CRF observed during training, a "preference" that could have produced a reversed PRE under circumstances where response-chain contingencies were not present (i.e., in the study by Pavlik \& Carlton, 1965). In any event, the results of the present experiments clearly indicate that studying the PRE within-subjects does not necessarily yield a reversed PRE, as our previous reports (Pavlik \& Carlton, 1965; Pavlik, Carlton, \& Hughes, 1965) have suggested. Rather, the nature of the PRE is a function of the particular contingencies employed. What kind of PRE, reversed or conventional, will prove to be the more general rule remains to be determined.

\section{References}

Pavlik, W. B., \& Carlton, P. L. A reversed partial reinforcement effect. J. exp. Psychol., 1965, 70, 417-423.

Pavlik, W. B., Carlton, P. L., \& Hughes, R. A. Partial reinforcement effects in a runway: Between- and within-Ss. Psychon. Sci., $1965,3,203-204$.

\section{Note}

1. The present research was supported by funds from USPHS Grant M-07844. 